\title{
On Bayesian One-sample Prediction of the Generalized Pareto Distribution based on Generalized Order Statistics
}

\author{
M.A.W. Mahmoud \\ Maths. Dept. Faculty of Science \\ Al-Azhar University \\ Nasr City 11884, Cairo, Egypt.
}

\author{
A.A.K. Saleh \\ Maths. Dept. Faculty of Women \\ Ain Shams University \\ Cairo, Egypt
}

\begin{abstract}
Bayesian predictive functions for future observations from a generalized Pareto distribution based on generalized order statistics are obtained. Two cases are considered unknown one parameter and unknown two parameters. We also consider two cases fixed sample size and random sample size. The Bayesian predictive functions are specialized to ordinary order statistics, progressive type II censoring and upper record values. Examples are calculated for the lower and the upper bounds for the future observation based on ordinary order statistics, progressive type II censoring and upper record samples..
\end{abstract}

MSC 2000: 60G25; 15A09; 62J05

\section{Keywords}

Bayesian prediction, generalized order statistics, generalized Pareto distribution, ordinary order statistics, predictive function, random sample size.

\section{INTRODUCTION}

The generalized Pareto distribution (GPD) was first introduced by [1]. The probability density function (pdf) and cumulative distribution function of the generalized Pareto are given by

$$
\begin{aligned}
& f(x ; \sigma, \theta)=\frac{1}{\sigma \theta}\left(1-\frac{x}{\sigma}\right)^{\frac{1}{\theta}-1} \quad ; \theta>0,0<x<\sigma,(1) \\
& \text { and } \quad F(x ; \sigma, \theta)=1-\left(1-\frac{x}{\sigma}\right)^{\frac{1}{\theta}}
\end{aligned}
$$

where $\theta$ is the shape parameter and $\sigma$ is the scale parameter. See [2].

Generalized order statistic's concept was presented by [3] as a unified approach to different models of ordered random variables such models are order statistics (OOS), record values (RVs), k-th record values and sequential order statistics, among others. Authors [4], [5] among others utilized the generalized order statistics (GOS) in their works. Let $X_{1}<X_{2}<\ldots<X_{r}$ be the first $r$ Generalized order failure times in a random sample of $n$ components from an absolutely continuous distribution function $F$, where $X_{i} \equiv X\left(i, n, m_{i}, k\right), i=1,2, \ldots, n, m_{i}$ and $k$ are real numbers and $k \geq 1$. The joint pdf of the first $r$ GOS is given by

$$
\begin{aligned}
f_{X_{1}, X_{2}, \ldots, X_{r}}\left(x_{1}, x_{2}, \ldots, x_{r}\right) & =C_{r-1}\left[\prod_{i=1}^{r-1}\left(1-F\left(x_{i}\right)\right)^{m_{i}} f\left(x_{i}\right)\right] \\
\times & {\left[\left(1-F\left(x_{r}\right)\right)^{\gamma_{r}-1} f\left(x_{r}\right)\right] }
\end{aligned}
$$

where $f\left(x_{i}\right)$ and $F\left(x_{i}\right)$ are the pdf and the cdf of $X_{i}$, and

$C_{j-1}=\prod_{i=1}^{j} \gamma_{i}, \quad \gamma_{i}=k+n-i+\sum_{j=i}^{n-1} m_{j}$ and $r=1,2, \ldots, n-1$.

See [3], considering two cases for the real numbers $m_{i}$, the conditional density function $h\left(x_{s} \mid x_{r}\right)$ of the GOS $x_{s}$ given that $r$ GOSs had already failed is given by,

Case I: When $m_{1}=m_{2}=\ldots=m_{n-1}=m=-1$

$$
\begin{aligned}
h_{1}\left(x_{s} \mid x_{r}\right) & =\frac{k^{s-r}}{(s-r-1) ! C_{r-1}}\left[\ln \left(1-F\left(x_{r}\right)\right)-\ln \left(1-F\left(x_{s}\right)\right)\right]^{s-r-1} \\
& \times\left[1-F\left(x_{s}\right)\right]^{k-1}\left[1-F\left(x_{r}\right)\right]^{-k} f\left(x_{s}\right),
\end{aligned}
$$

Case II: When $m_{i} \neq m_{j}$ for $i \neq j$,

$$
\begin{aligned}
h_{2}\left(x_{s} \mid x_{r}\right) & =\frac{C_{s-1}}{C_{r-1}} \\
& \times \sum_{i=r+1}^{s} a_{i}^{(r)}(s)\left(\frac{1-F\left(x_{s}\right)}{1-F\left(x_{r}\right)}\right)^{\gamma_{i}}\left(\frac{f\left(x_{s}\right)}{1-F\left(x_{s}\right)}\right), 1 \leq r \leq n-1,
\end{aligned}
$$

where

$$
a_{i}^{(r)}(s)=\prod_{\substack{j=r+1 \\ j \neq i}}^{s} \frac{1}{\gamma_{j}-\gamma_{i}}, \quad r+1 \leq i \leq s .
$$

See [3], [6]. The Bayes prediction of an unobserved random variable is frequently more significant for applications than interval estimation of parameters. Many authors have been discussed the prediction problems in general based on GOS, progressively type-II censored data, record values (RVs) and ordinary order statistics (OOS) see for example [7], [8]. The goal of this paper is to discuss the one-sample Bayesian prediction problem from generalized Pareto distribution based on GOS in two cases when the sample size is fixed (FSS) and when it is a random variable $(R S S)$. In case of fixed sample size, The Bayes predictive density function of the future GOS $x_{s}, s=r+1, r+2, \ldots, n$ can be written as:

$$
f^{*}\left(x_{s} \mid \underline{x}\right)=\int_{\sigma \theta} \int_{\theta} h\left(x_{s} \mid \theta, \sigma\right) f(\theta, \sigma \mid \underline{x}) d \theta d \sigma,
$$

where the conditional pdf of the future observation is $h\left(x_{s} \mid \theta, \sigma\right)$ and $f(\theta, \sigma \mid \underline{x})$ is the posterior pdf.

In case of random sample size, the predictive density function 


$$
\begin{aligned}
& \text { of } \\
& x_{s} \\
& \text { is } \\
& \text { given }
\end{aligned}
$$

where $v(n)$ is the probability mass function of $n$ and $f^{*}\left(x_{s} \mid \underline{x}\right)$ is defined in (8), see for example [9].

\section{BAYESIAN PREDICTION BOUNDS BASED ON GOS IN CASE OF FSS}

The likelihood function of the first $r$ GOS failure times $x_{1}, x_{2}, \ldots, x_{r}$ in a random sample of $n$ identical distributed random variables having the GPD defined in (1) can be obtained by substituting (1) and (2) into (3) as follows

$$
L(\sigma, \theta ; x)=C_{r-1}(\sigma \theta)^{-r} \eta(\sigma) e^{\frac{T}{\theta}},
$$

where

$$
\eta(\sigma)=\prod_{i=1}^{i=r}\left(1-\frac{x_{i}}{\sigma}\right)^{-1}, T=\sum_{i=1}^{i=r-1}\left(m_{i}+1\right) \ln \left(1-\frac{x_{i}}{\sigma}\right)+\gamma_{r} \ln \left(1-\frac{x_{r}}{\sigma}\right)
$$

Considering that the scale parameters $\sigma$ is known and the unknown shape parameter $\theta$ is having the inverted gamma prior distribution defined by

$$
g_{1}(\theta)=\frac{\beta^{\alpha}}{\Gamma(\alpha)} \theta^{-(\alpha+1)} e^{-\frac{\beta}{\theta}}, \quad \text { if } \theta>0,(\alpha, \beta),
$$

the posterior pdf of $\theta$ can be written as

$$
f_{1}\left(\theta \mid \underset{-}{x)}=\frac{(\beta-T)^{\alpha+r}}{\Gamma(\alpha+r)} \theta^{-(r+\alpha+1)} e^{-\frac{\beta-T}{\theta}},\right.
$$

when the two parameters $\sigma$ and $\theta$ are assumed to be unknown, the inverted gamma prior density defined in (12) is considered as a distribution for the unknown shape parameter $\theta$ and the uniform prior density is considered for the unknown scale parameters $\sigma$ defined by

$$
g_{2}(\sigma)=\frac{1}{v}, \quad 0 \leq \sigma \leq v,
$$

the joint prior density for both the parameters $\theta$ and $\sigma$ takes the form

$$
g(\sigma, \theta)=\frac{\beta^{\alpha}}{\Gamma(\alpha)} \theta^{-(\alpha+1)} e^{-\frac{\beta}{\theta}} \frac{1}{v}, 0 \leq \sigma \leq v,(\alpha, \beta, \theta, v)>0,
$$

where $\alpha, \beta$, and $v$ are known. The joint posterior pdf of $\theta$ and $\sigma$ is given by

$$
f_{2}(\sigma, \theta \mid \underline{x})=\frac{\eta(\sigma)}{\Gamma(\alpha+r) \sigma^{r} I_{0}} \theta^{-(r+\alpha+1)} e^{-\frac{\beta-T}{\theta}}
$$

where $I_{0}=\int_{0}^{v} \frac{\eta(\sigma)}{\sigma^{r}}(\beta-T)^{-(r+\alpha)} d \sigma$.

\subsection{When $\sigma$ is known}

Now let $x_{s}, s=r+1, r+2, \ldots, n$ denotes the $s^{\text {th }}$ lifetime component from the remaining $(n-r)$ components having the GPD when the scale parameter $\sigma$ is known, the conditional density function of the $s^{\text {th }}$ GOS given that $r$ generalized order statistics had already failed can be obtained by substituting (1) and (2) into (5) and (6) as follows:

Case

$h_{1}\left(x_{s} \mid x_{r}\right)=\frac{k^{s-r}}{\sigma \Gamma(s-r)}\left(\frac{1}{\theta}\right)^{s-r}\left(1-\frac{x_{s}}{\sigma}\right)^{-1} A^{s-r-1} e^{-\frac{A k}{\theta}}$

Case

II:

$h_{2}\left(x_{s} \mid x_{r}\right)=\frac{C_{s-1}}{\sigma C_{r-1}}\left(1-\frac{x_{s}}{\sigma}\right)^{-1} \sum_{i=r+1}^{s} a_{i}^{(r)}(s) \frac{1}{\theta} e^{A \frac{\gamma_{i}}{\theta}}$,

where $\quad A=-\ln \left[\frac{\sigma-x_{s}}{\sigma-x_{r}}\right]$.

The Bayes predictive density function of the future GOS $x_{s}$ is obtained by substituting (18), (19) and (13) into (8)

Case I:

$$
\begin{aligned}
f_{11}^{*}\left(x_{s} \mid \underline{x}\right)= & \frac{k^{s-r}}{\sigma} \frac{\left(\beta-k T_{r}\right)^{\alpha+r} \Gamma(\alpha+s)}{\Gamma(s-r) \Gamma(\alpha+r)} \\
& \times \frac{\left(1-\frac{x_{s}}{\sigma}\right)^{-1} A^{s-r-1}}{\left(\beta-k \ln \left(1-\frac{x_{s}}{\sigma}\right)\right)^{\alpha+s}} .
\end{aligned}
$$

Case II:

$$
\begin{aligned}
& f_{12}^{*}\left(x_{s} \mid \underline{x}\right)= \frac{(\alpha+r) C_{s-1}}{C_{r-1}}\left(\sigma-x_{s}\right)^{-1} \\
& \sum_{i=r+1}^{s} \frac{a_{i}^{(r)}(s)(\beta-T)^{\alpha+r}}{\left(\beta-T+A \gamma_{i}\right)^{\alpha+r+1}} .
\end{aligned}
$$

By integrating with respect to $x_{s}$, the Bayesian prediction survival function $\operatorname{Pr}\left(x_{s}>t \mid \underline{x}\right)$ for some $t$, can be written as follows:

Case

$$
\begin{aligned}
& \operatorname{Pr}_{1}\left(x_{s} \geq t \mid \underline{x}\right)=R_{r, s}\left[\frac{1}{(\alpha+s-1)} \frac{\left(k T_{r}-k Z\right)^{s-r-1}}{(\beta-k Z)^{\alpha+s-1}}\right. \\
& \quad+\frac{s-r-1}{(\alpha+s-1)(\alpha+s-2)} \frac{\left(k T_{r}-k Z\right)^{s-r-2}}{(\beta-k Z)^{\alpha+s-2}} \\
& +\frac{(s-r-1)(s-r-2)}{(\alpha+s-1)(\alpha+s-2)(\alpha+s-3)} \frac{\left(k T_{r}-k Z\right)^{s-r-3}}{(\beta-k Z)^{\alpha+s-3}}+\ldots \\
& \left.+\frac{(s-r-1) !}{(\alpha+s-1)(\alpha+s-2) \ldots(\alpha+r)} \frac{1}{(\beta-k Z)^{\alpha+r}}\right],(23)
\end{aligned}
$$

Case II:

$$
\begin{aligned}
\operatorname{Pr}_{2}\left(x_{s} \geq t \mid \underline{x}\right)=\frac{C_{s-1}}{C_{r-1}}(\beta-T)^{\alpha+r} \\
\quad \times \sum_{i=r+1}^{s} \frac{a_{i}^{(r)}(s)}{\gamma_{i}}\left[\beta-T-\gamma_{i}\left(Z-T_{r}\right)\right]^{-(. \alpha+r)},
\end{aligned}
$$

where $T_{r}=\ln \left(1-\frac{x_{r}}{\sigma}\right), Z=\ln \left(1-\frac{t}{\sigma}\right)$, 
and $\quad R_{r, s}=\frac{\left(\beta-T_{r}\right)^{\alpha+r} \Gamma(\alpha+s)}{\Gamma(s-r) \Gamma(\alpha+r)}$.

Let $L_{s}$ and $U_{s}$ denote the lower and the upper limits of the $100 \tau \%$ Bayesian prediction interval for the GOS $x_{s}$ such that $\operatorname{pr}\left[L_{s} \leq x_{s} \leq U_{s}\right]=\tau$ where,

$$
\operatorname{Pr}\left(x_{s} \geq L_{s} \mid \underline{x}\right)=\frac{1+\tau}{2}, \operatorname{Pr}\left(x_{s} \geq U_{s} \mid \underline{x}\right)=\frac{1-\tau}{2} .
$$

\subsection{When $\theta$ and $\sigma$ are Unknown parameters} In this subsection, The Bayes predictive density function of the GOS $x_{s}$ is obtained by substituting (18), (19) and (16) into (8):

Case I:

$$
\begin{aligned}
f_{21}^{*}\left(x_{s} \mid \underline{\mathrm{x}}\right) & =\frac{k^{s-r}}{I_{0} \Gamma(s-r)} \frac{\Gamma(\alpha+s)}{\Gamma(\alpha+r)} \\
& \int_{0}^{v} \frac{\eta(\sigma)\left(1-\frac{x_{s}}{\sigma}\right)^{-1} A^{s-r-1}}{\left(\beta-k \ln \left(1-\frac{x_{s}}{\sigma}\right)\right)^{\alpha+s} \sigma^{r+1}} d \sigma .
\end{aligned}
$$

Case II:

$$
\begin{aligned}
& f_{22}{ }^{*}\left(x_{s} \mid \underline{x}\right)=\frac{(\alpha+r) C_{s-1}}{I_{0} C_{r-1}} \\
& \int_{0}^{v} \frac{\eta(\sigma)}{\sigma^{r}\left(\sigma-x_{s}\right)} \sum_{i=r+1}^{s} \frac{a_{i}^{(r)}(s)}{\left(\beta-T+A \gamma_{i}\right)^{(\alpha+r+1)}} d \sigma,
\end{aligned}
$$

and the Bayesian prediction survival function of the future GOS $x_{s}$ as follows:

CaseI:

$$
\begin{gathered}
\operatorname{Pr}_{21}\left(x_{s} \geq t \mid \underline{x}\right)=\frac{\Gamma(\alpha+s)}{I_{0} \Gamma(\alpha+r) \Gamma(s-r)} \\
\quad \times \int_{0}^{v} \frac{\eta(\sigma)}{\sigma^{r}}\left[\frac{1}{(\alpha+s-1)} \frac{\left(k T_{r}-k Z\right)^{s-r-1}}{(\beta-k Z)^{\alpha+s-1}}\right. \\
+\frac{s-r-1}{(\alpha+s-1)(\alpha+s-2)} \frac{\left(k T_{r}-k Z\right)^{s-r-2}}{(\beta-k Z)^{\alpha+s-2}} \\
+\frac{(s-r-1)(s-r-2)}{(\alpha+s-1)(\alpha+s-2)(\alpha+s-3)} \frac{\left(k T_{r}-k Z\right)^{s-r-3}}{(\beta-k Z)^{\alpha+s-3}}+\ldots \\
\left.+\frac{(s-r-1) !}{(\alpha+s-1)(\alpha+s-2) \ldots(\alpha+r)} \frac{1}{(\beta-k Z)^{\alpha+r}}\right] d \sigma,(30)
\end{gathered}
$$

CaseII:

$$
\begin{aligned}
\operatorname{Pr}_{22}\left(x_{s} \geq t \mid \underline{x}\right)=\frac{C_{s-1}}{I_{0} C_{r-1}} \sum_{i=r+1}^{s} a_{i}^{(r)}(s) \\
\quad \times \int_{0}^{v} \frac{\eta(\sigma)}{\gamma_{i} \sigma^{r}}\left[\beta-T-\gamma_{i}\left(Z-T_{r}\right)\right]^{-(\alpha+r) .} .
\end{aligned}
$$

\section{BAYESIAN PREDICTION BOUNDS BASED ON GOS IN CASE OF RSS}

In this section, assuming that the sample size $n$ is a random variable distributed as a Poisson distribution with pmf given by

$$
p(n ; \lambda)=\frac{e^{-\lambda} \lambda^{n}}{n !}, \quad n=0,1,2, \ldots \text { and } \lambda>0 .
$$

Replacing $v(n)$ in (9) by $p(n ; \lambda)$ defined in (32), we get

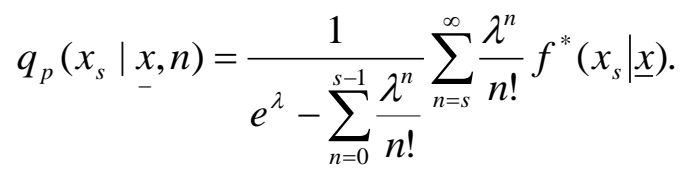

\subsection{When $\sigma$ is known}

Assuming that $\sigma$ is known, substituting (21) and (22) into (33), the bayes predictive functions take the forms:

Case I:

$$
\begin{gathered}
q_{p 11}\left(x_{s} \mid \underline{x}, n\right)=\frac{\Gamma(\alpha+s)\left(\sigma-x_{s}\right)^{-1}}{\Gamma(\alpha+r) \Gamma(s-r)\left[e^{\lambda}-\sum_{n=0}^{s-1} \frac{\lambda^{n}}{n !}\right]} \\
\times \sum_{n=s}^{\infty} \frac{\lambda^{n}}{n !} \frac{k^{s-r}\left(\beta-k T_{r}\right)^{\alpha+r} A^{s-r-1}}{\left(\beta-k \ln \left(1-\frac{x_{s}}{\sigma}\right)\right)^{\alpha+s}},
\end{gathered}
$$

Case II:

$$
\begin{aligned}
& q_{p 12}\left(x_{s} \mid \underline{x}, n\right)=\frac{(\alpha+r)}{e^{\lambda}-\sum_{n=0}^{s-1} \frac{\lambda^{n}}{n !}}\left(\sigma-x_{s}\right)^{-1} \\
& \times \sum_{n=s}^{\infty} \sum_{i=r+1}^{s} \frac{\lambda^{n}}{n !} \frac{C_{s-1}}{C_{r-1}} a_{i}^{(r)}(s) \frac{(\beta-T)^{\alpha+r}}{\left(\beta-T+A \gamma_{i}\right)^{(\alpha+r+1)}},
\end{aligned}
$$

and hence integrating (34) and (35) with respect to $x_{s}$ (from some $t$ to $\sigma$ ), the Bayesian prediction survival function is obtained:

\section{CaseI:}

$$
\begin{aligned}
& \operatorname{Pr}_{p 11}\left(x_{s} \geq t \mid \underline{x}\right)= \\
& \frac{1}{e^{\lambda}-\sum_{n=0}^{s-1} \frac{\lambda^{n}}{n !} \sum_{n=s}^{\infty}\left[\frac { \lambda ^ { n } } { n ! } R _ { r , s } \left[\frac{1}{(\alpha+s-1)} \frac{\left(k T_{r}-k Z\right)^{s-r-1}}{(\beta-k Z)^{\alpha+s-1}}\right.\right.} \\
& \quad+\frac{s-r-1}{(\alpha+s-1)(\alpha+s-2)} \frac{\left(k T_{r}-k Z\right)^{s-r-2}}{(\beta-k Z)^{\alpha+s-2}} \\
& +\frac{(s-r-1)(s-r-2)}{(\alpha+s-1)(\alpha+s-2)(\alpha+s-3)} \frac{\left(k T_{r}-k Z\right)^{s-r-3}}{(\beta-k Z)^{\alpha+s-3}} \\
& \left.\left.+\ldots+\frac{(s-r-1) !}{(\alpha+s-1)(\alpha+s-2) \ldots(\alpha+r)} \frac{1}{(\beta-k Z)^{\alpha+r}}\right]\right],
\end{aligned}
$$


CaseII:

$$
\begin{gathered}
\operatorname{Pr}_{p 12}\left(x_{s} \geq t \mid \underline{x}\right)=\frac{1}{e^{\lambda}-\sum_{n=0}^{s-1} \frac{\lambda^{n}}{n !}} \\
\sum_{n=s}^{\infty} \sum_{i=r+1}^{s} \frac{\lambda^{n} C_{s-1}}{n ! C_{r-1}} \frac{a_{i}^{(r)}(s)(\beta-T)^{\alpha+r}}{\gamma_{i}\left[\beta-T-\gamma_{i}\left(Z-T_{r}\right)\right]^{(\alpha+r)}} .
\end{gathered}
$$

\subsection{When $\theta$ and $\sigma$ are Unknown parameters}

When the two parameters having posterior pdf given by (16) and the sample size $n$ has a Poisson distribution with pmf given by (32), The Bayes predictive density function of the GOS $x_{s}$ is obtained by substituting (28) and (29) into (33) as follows:

\section{CaseI:}

$$
\begin{aligned}
& q_{p 21}\left(x_{s} \mid \underline{x}, n\right)=\frac{\Gamma(\alpha+s)}{I_{0} \Gamma(\alpha+r) \Gamma(s-r)} \frac{k^{s-r}}{e^{\lambda}-\sum_{n=0}^{s-1} \frac{\lambda^{n}}{n !}} \\
& \sum_{n=s}^{\infty} \frac{\lambda^{n}}{n !} \int_{0}^{v} \frac{\eta(\sigma)\left(\sigma-x_{s}\right)^{-1} A^{s-r-1}}{\sigma^{r}\left(\beta-k \ln \left(1-\frac{x_{s}}{\sigma}\right)\right)^{\alpha+s}} d \sigma
\end{aligned}
$$

Case II:

$$
\begin{aligned}
q_{p 22}\left(x_{s} \mid \underline{x}, n\right) & =\frac{(\alpha+r)}{e^{\lambda}-\sum_{n=0}^{s-1} \frac{\lambda^{n}}{n !} \sum_{n=s}^{\infty} \sum_{i=r+1}^{s} \frac{\lambda^{n}}{n !} \frac{a_{i}^{(r)}(s) C_{s-1}}{I_{0} C_{r-1}}} \\
& \int_{0}^{v} \frac{\eta(\sigma)\left(\sigma-x_{s}\right)^{-1}}{\sigma^{r}\left(\beta-T+A \gamma_{i}\right)^{(\alpha+r+1)}} d \sigma
\end{aligned}
$$

and the Bayesian prediction survival function of the future GOS $x_{s}, s=r+1, r+2, \ldots, n$ in this case take the forms:

CaseI:

$$
\begin{aligned}
& \operatorname{Pr}_{p 21}\left(x_{s} \geq t \mid \underline{x}\right)= \\
& \frac{\Gamma(\alpha+s)}{I_{0} \Gamma(\alpha+r) \Gamma(s-r)} \frac{1}{e^{\lambda}-\sum_{n=0}^{s-1} \frac{\lambda^{n}}{n !}} \\
& \times \sum_{n=s}^{\infty} \frac{\lambda^{n}}{n !} \int_{0}^{v}\left[\frac { \eta ( \sigma ) } { \sigma ^ { r } } \left[\frac{1}{(\alpha+s-1)} \frac{\left(k T_{r}-k Z\right)^{s-r-1}}{(\beta-k Z)^{\alpha+s-1}}\right.\right. \\
& +\frac{s-r-1}{(\alpha+s-1)(\alpha+s-2)} \frac{\left(k T_{r}-k Z\right)^{s-r-2}}{(\beta-k Z)^{\alpha+s-2}} \\
& +\frac{(s-r-1)(s-r-2)}{(\alpha+s-1)(\alpha+s-2)(\alpha+s-3)} \frac{\left(k T_{r}-k Z\right)^{s-r-3}}{(\beta-k Z)^{\alpha+s-3}}+\ldots \\
& \left.\left.+\frac{(s-r-1) !}{(\alpha+s-1)(\alpha+s-2) \ldots(\alpha+r)} \frac{1}{(\beta-k Z)^{\alpha+r}}\right]\right] d \sigma,(40)
\end{aligned}
$$

Case II:

$$
\begin{gathered}
\operatorname{Pr}_{p 22}\left(x_{s} \geq t \mid \underline{x}\right)=\frac{1}{e^{\lambda}-\sum_{n=0}^{s-1} \frac{\lambda^{n}}{n !} \sum_{n=s}^{\infty} \sum_{i=r+1}^{s} \frac{a_{i}^{(r)}(s) C_{s-1}}{I_{0} C_{r-1}} \frac{\lambda^{n}}{n !}} \\
\quad \times \int_{0}^{v} \frac{\eta(\sigma)}{\gamma_{i} \sigma^{r}}\left[\beta-T-\gamma_{i}\left(Z-T_{r}\right)\right]^{-(\alpha+r)} d \sigma,
\end{gathered}
$$

\section{BAYESIAN PREDICTION BASED ON UPPER RVS IN CASE OF FSS}

It is well known that the record value is a GOS when $k=1$, and $m=-1$.

\subsection{When $\sigma$ is known}

The Bayes predictive density function of the future upper RVs $x_{s}, s=r+1, r+2, \ldots, n$, is given by putting $(k=1, m=-1)$ in (21).

$$
\begin{aligned}
f_{R V 1}^{*}\left(x_{s} \mid \underline{x}\right)= & \frac{\Gamma(\alpha+s)}{\sigma \Gamma(s-r) \Gamma(\alpha+r)}\left(\beta-T_{r}\right)^{\alpha+r} \\
& \frac{\left(1-\frac{x_{s}}{\sigma}\right)^{-1} A^{s-r-1}}{\left(\beta-\ln \left(1-\frac{x_{s}}{\sigma}\right)\right)^{\alpha+s}}
\end{aligned}
$$

the Bayesian prediction survival function of the upper RVs $x_{s}$ can be obtained from (23) as follows:

$$
\begin{aligned}
& \operatorname{Pr}_{R V 1}\left(x_{s} \geq t \mid \underline{x}\right)= \\
& \frac{\Gamma(\alpha+s)\left(\beta-T_{r}\right)^{\alpha+r}}{\Gamma(s-r) \Gamma(\alpha+r)}\left[\frac{1}{(\alpha+s-1)} \frac{\left(T_{r}-Z\right)^{s-r-1}}{(\beta-Z)^{\alpha+s-1}}\right. \\
& +\frac{s-r-1}{(\alpha+s-1)(\alpha+s-2)} \frac{\left(T_{r}-Z\right)^{s-r-2}}{(\beta-Z)^{\alpha+s-2}} \\
& +\frac{(s-r-1)(s-r-2)}{(\alpha+s-1)(\alpha+s-2)(\alpha+s-3)} \frac{\left(T_{r}-Z\right)^{s-r-3}}{(\beta-Z)^{\alpha+s-3}}+\ldots \\
& \left.+\frac{(s-r-1) !}{(\alpha+s-1)(\alpha+s-2) \ldots(\alpha+r)} \frac{1}{(\beta-Z)^{\alpha+r}}\right] .(43)
\end{aligned}
$$

The lower and the upper limits of the $100_{\tau} \%$ Bayesian prediction interval for the future upper RV $x_{s}$ are obtained by substituting (43) into (27).

\subsection{When $\theta$ and $\sigma$ are Unknown parameters}

When the two parameters are unknown and having joint prior pdf given by (16), putting $(k=1, m=-1)$ in (28) and (30) the Bayes predictive density function and the Bayesian prediction survival function of the upper RVs $x_{s}$ are obtained as follows:

$$
\begin{aligned}
f_{R V 2}^{*}\left(x_{s} \mid \underline{x}\right) & =\frac{1}{I_{1}} \frac{\Gamma(\alpha+s)}{\Gamma(s-r) \Gamma(\alpha+r)} \\
& \times \int_{0}^{v} \frac{\eta(\sigma)\left(1-\frac{x_{s}}{\sigma}\right)^{-1} A^{s-r-1}}{\left(\beta-\ln \left(1-\frac{x_{s}}{\sigma}\right)\right)^{\alpha+s} \sigma^{r+1}} d \sigma,
\end{aligned}
$$


$\operatorname{Pr}_{R V 2}\left(X_{s} \geq t \mid \underline{x}\right)=\frac{\Gamma(\alpha+s)}{I_{1} \Gamma(\alpha+r) \Gamma(s-r)}$

$\times \int_{0}^{v} \frac{\eta(\sigma)}{\sigma^{r}}\left[\frac{1}{(\alpha+s-1)} \frac{\left(T_{r}-Z\right)^{s-r-1}}{(\beta-Z)^{\alpha+s-1}}\right.$

$+\frac{s-r-1}{(\alpha+s-1)(\alpha+s-2)} \frac{\left(T_{r}-Z\right)^{s-r-2}}{(\beta-Z)^{\alpha+s-2}}$

$+\frac{(s-r-1)(s-r-2)}{(\alpha+s-1)(\alpha+s-2)(\alpha+s-3)} \frac{\left(T_{r}-Z\right)^{s-r-3}}{(\beta-Z)^{\alpha+s-3}}+\ldots$

$\left.+\frac{(s-r-1) !}{(\alpha+s-1)(\alpha+s-2) \ldots(\alpha+r)} \frac{1}{(\beta-Z)^{\alpha+r}}\right] d \sigma,(45)$

where $\quad I_{1}=\int_{0}^{v} \frac{\eta(\sigma)}{\sigma^{r}}\left(\beta-T_{r}\right)^{-(r+\alpha)} d \sigma$.

\section{BAYESIAN PREDICTION BASED ON UPPER RVS IN CASE OF RSS}

It is clear that the equations obtained in Section 4 are all independent of the sample size $n$.

\subsection{When $\sigma$ is known}

Assuming that the sample size is a random variable with the probability mass function $v(n)$, substituting by (42) into (9), the Bayes predictive density function is obtained as follows:

$$
\begin{gathered}
q_{R V 1}\left(x_{s} \mid \underline{x}, n\right)=\frac{1}{\operatorname{Pr}(n \geq s)} \sum_{n=s}^{\infty} v(n) f_{R V 1}^{*}\left(x_{s} \mid \underline{x}\right) \\
=\frac{f_{R V 1}^{*}\left(x_{s} \mid \underline{x}\right)}{\operatorname{Pr}(n \geq s)} \sum_{n=s}^{\infty} v(n) \\
=\frac{f_{R V 1}^{*}\left(x_{s} \mid \underline{x}\right)}{\operatorname{Pr}(n \geq s)} \operatorname{Pr}(n \geq s)=f_{R V 1}^{*}\left(x_{s} \mid \underline{x}\right)
\end{gathered}
$$

Similarly, the survival function of the upper RV $x_{s}$ has the same formulae of equation (43) weather the sample size is fixed or random variable.

\subsection{When $\theta$ and $\sigma$ are Unknown parameters}

Similarly, the Bayes predictive density function and the survival function of the upper RV $x_{s}$, can be obtained from equations (44) and (45) respectively.

\section{BAYESIAN PREDICTION BASED ON PROGRESSIVELY TYPE-II CENSORED ORDER STATISTICS IN CASE OF FSS}

Let $x_{1: \ell: n}, x_{2: \ell: n}, \ldots, x_{\ell: \ell: n}$ denote a progressively Type-II censored sample, from GPD defined in (1) and (2), with censoring scheme $\underline{R}=\left(R_{1}, R_{2}, \ldots, R_{\ell}\right)$ then the conditional density function of the future progressively Type-II censored order statistic $x_{s}$ can be obtained by putting $m_{i}=R_{i}$ for $1 \leq i \leq \ell-1, \quad \ell \in\{1,2, \ldots, n-1\}$ and $\gamma_{\ell}=k=R_{\ell}+1$ in (19), for more details see [10] and [11].

\subsection{When $\sigma$ is known}

The Bayes predictive density function and the Bayesian prediction survival function in this case will take the forms:

$$
\begin{gathered}
f_{p r o g 1}^{*}\left(x_{s} \mid \underline{x}\right)=\frac{C_{s-1}}{\sigma C_{r-1}}(\alpha+r)\left(1-\frac{x_{s}}{\sigma}\right)^{-1} \\
\sum_{i=r+1}^{s} \frac{a_{i}^{*(r)}(s)\left(\beta-T_{R}\right)^{\alpha+r}}{\left(\beta-T_{R}-A \delta_{i}\right)^{(\alpha+r+1)}}
\end{gathered}
$$

and

$$
\begin{aligned}
& \operatorname{Pr}_{\text {progl }}\left(x_{s} \geq t \mid \underline{x}\right)=\prod_{k=r+1}^{s} \delta_{k}\left(\beta-T_{R}\right)^{\alpha+r} \\
& \sum_{i=r+1}^{s} a_{i}^{*(r)}(s) \frac{1}{\delta_{i}}\left[\beta-T_{R}-\delta_{i} \ln \left[\frac{\sigma-t}{\sigma-x_{r}}\right]\right]^{-(\alpha+r)},
\end{aligned}
$$

where

$$
\begin{gathered}
T_{R}=\sum_{i=1}^{i=r-1}\left(R_{i}+1\right) \ln \left(1-\frac{x_{i}}{\sigma}\right)+\delta_{r} \ln \left(1-\frac{x_{r}}{\sigma}\right) \\
\delta_{i}=\sum_{j=i}^{\ell}\left(R_{j}+1\right)=n-\sum_{j=1}^{i-1}\left(R_{j}+1\right), \\
\text { and } a_{i}^{*(r)}(s)=\prod_{\substack{j=r+1 \\
j \neq i}}^{s} \frac{1}{\delta_{j}-\delta_{i}}, r+1 \leq i \leq s .
\end{gathered}
$$

6.2 When $\theta$ and $\sigma$ are Unknown parameters The Bayes predictive density function and the Bayesian prediction survival function of the future progressively type-II censored $x_{s}, s=r+1, r+2, \ldots, n$ will take the forms

$$
\begin{array}{r}
f_{p r o 2}^{*}\left(x_{s} \mid \underline{x}\right)=\frac{C_{s-1}}{I_{3} C_{r-1}} \int_{0}^{v} \frac{\eta(\sigma ; \underline{x})}{\sigma^{r}\left(\sigma-x_{s}\right)} \\
\sum_{i=r+1}^{s} \frac{a_{i}^{*(r)}(s)(\alpha+r)}{\left(\beta-T_{R}-A \delta_{i}\right)^{(\alpha+r+1)}} d \sigma,
\end{array}
$$

and

$$
\begin{aligned}
\operatorname{Pr}_{\text {pro } 2}\left(x_{s} \geq t \mid \underline{x}\right)=\frac{C_{s-1}}{I_{3} C_{r-1}} \\
\sum_{i=r+1}^{s} \int_{0}^{v} \frac{a_{i}^{*(r)}(s) \eta(\sigma ; \underline{x})}{\sigma^{r} \delta_{i}\left[\beta-T_{R}-\delta_{i}\left(Z-T^{\prime \prime}\right)\right]^{(\alpha+r)}} d \sigma .
\end{aligned}
$$

Where $I_{3}=\int_{0}^{v} \frac{\eta(\sigma)}{\sigma^{r}}\left(\beta-T_{R}\right)^{-(r+\alpha)} d \sigma$.

\section{BAYESIAN PREDICTION BASED ON PROGRESSIVELY TYPE-II CENSORED ORDER STATISTICS IN CASE OF RSS}

\subsection{When $\sigma$ is known}

The Bayes predictive density function and the Bayesian prediction survival function will take the forms 


$$
\begin{aligned}
& q_{\text {prolP }}\left(x_{s} \mid \underline{x}, n\right)=\frac{(\alpha+r)}{e^{\lambda}-\sum_{n=0}^{s-1} \frac{\lambda^{n}}{n !}}\left(\sigma-x_{s}\right)^{-1} \\
& \times \sum_{n=s}^{\infty} \sum_{i=r+1}^{s} \frac{\lambda^{n}}{n !} \frac{C_{s-1}}{C_{r-1}} a_{i}^{*(r)}(s) \frac{\left(\beta-T_{R}\right)^{\alpha+r}}{\left(\beta-T_{R}-A \delta_{i}\right)^{(\alpha+r+1)}},
\end{aligned}
$$

and

$$
\begin{aligned}
& \operatorname{Pr}_{p r o 1 P}\left(x_{s} \geq t \mid \underline{x}\right)=\frac{1}{e^{\lambda}-\sum_{n=0}^{s-1} \frac{\lambda^{n}}{n !}} \\
& \sum_{n=s}^{\infty} \sum_{i=r+1}^{s} \frac{\lambda^{n} C_{s-1}}{n ! C_{r-1}} \frac{a_{i}^{*(r)}(s)\left(\beta-T_{R}\right)^{\alpha+r}}{\delta_{i}\left[\beta-T_{R}-\delta_{i}\left(Z-T^{\prime \prime}\right)\right]^{(\alpha+r)}} .
\end{aligned}
$$

\subsection{When $\theta$ and $\sigma$ are Unknown parameters}

The Bayes predictive density function and the Bayesian prediction survival function of the future progressively type-II censored $\mathrm{OS} x_{s}, s=r+1, r+2, \ldots, n$ in this case take the forms

$$
\begin{aligned}
& q_{\text {pro2P }}\left(x_{s} \mid \underset{-}{x, n)}=\frac{(\alpha+r)}{e^{\lambda}-\sum_{n=0}^{s-1} \frac{\lambda^{n}}{n !}}\right. \\
& \sum_{n=s}^{\infty} \sum_{i=r+1}^{s} \frac{\lambda^{n}}{n !} \frac{a_{i}^{*(r)}(s) C_{s-1}}{I_{R} C_{r-1}} \int_{0}^{v} \frac{\eta(\sigma ; \underline{x})\left(\sigma-x_{s}\right)^{-1} \sigma^{-r}}{\left(\beta-T_{R}-A \delta_{i}\right)^{(\alpha+r+1)}} d \sigma,
\end{aligned}
$$

and

$$
\begin{aligned}
& \operatorname{Pr}_{p r o 2 P}\left(x_{s} \geq t \mid \underline{x}\right)=\frac{1}{e^{\lambda}-\sum_{n=0}^{s-1} \frac{\lambda^{n}}{n !}} \\
& \times \sum_{n=s}^{\infty} \sum_{i=r+1}^{s} \frac{a_{i}^{*}(r)}{I_{3} C_{r-1}} \frac{\lambda_{s-1}}{n !} \\
& \int_{0}^{v} \frac{\eta(\sigma ; \underline{x})}{\gamma_{i} \sigma^{r}}\left[\beta-T_{R}-\delta_{i}\left(Z-T^{\prime \prime}\right)\right]^{-(\alpha+r)} d \sigma
\end{aligned}
$$

\section{10. Numerical analysis}

In this section, Bayesian prediction bounds for the $s^{\text {th }}$ observation in samples of progressively type-II censored and upper record sample with fixed and random sample sizes are obtained.

\subsection{Unknown one parameter}

By taking the parameter $\sigma$ is known $(\sigma=0.1$ and 0.5$)$, one can find the $90 \%$ and $95 \%$ BPI for the future observation $x_{s: n}$ using Monte Carlo simulation study according to the following steps:

1. For arbitrary given values of the prior parameters $(\alpha, \beta)=(1,0.1)$, we generate $\theta$ using (12).

2. According to an algorithm introduced by [12], two progressively type -II censored samples with given censoring scheme $(1,0,2,1,0,3,0,2,0,1)$ from GPD with $\alpha, \beta$ and $\sigma$ as in step (1) and (2) are generated $\{0.0003,0.0006,0.0007,0.0025,0.0036$, $0.004,0.0085,0.0117,0.0138,0.0295\}$ and
$\{0.00017,0.00019,0.0002,0.0005,0.0006,0.0008$, $0.0013,0.0015,0.0021,0.0021\}$. Taking the number of observed failures is $r=7$ and 9 , the $90 \%$ and $95 \%$ BPI for the future $x_{s: n}, s=8,9$ and 10 by evaluating (49) with (27) are constructed, these are given in Table 1.

3. Using generated $\theta$ obtained as in step (1), two samples of upper records from GPD given in (1) with $\sigma=0.1$ and 0.5 and fixed sample size $n=10$ are generated, $\{0.0204,0.0239,0.0329,0.1644$, $0.1882,0.1965,0.2698,0.2870,0.3026,0.3421\}$ and $\{0.0332, \quad 0.0462,0.0562,0.0618,0.0629$, $0.0689,0.0690,0.0712,0.0715,0.0745\}$. Taking the number of observed upper records is $\mathrm{r}=7$ and 8 , $90 \%$ and $95 \%$ BPI for the future observation $x_{s: n}$, $s=8,9$ and 10 are constructed by evaluating (43) with (27), noting that the results doesn't depend on the sample size $n$. Table 3 represents these results.

4. Repeat step (4) with initial sample size $n=7$ from Poisson distribution with $\lambda=0.9$, two progressively type II censoring samples are generated. The constructed $90 \%$ and $95 \%$ BPI for the future $x_{s: n}$, $s=8,9$ and 10 by evaluating (55) with (27) are in Table 2.

\subsection{Unknown two parameters}

Assuming that the parameters $\theta$ and $\sigma$ are unknown, the $90 \%$ and $95 \%$ BPI for the future observation $x_{s: n}$ using the Monte Carlo simulation study are computed according to the following steps:

1. $90 \%$ and $95 \%$ BPI for the future upper RVs observation $x_{s: n}, s=8,9$ and 10 are obtained from two different samples in Table 4.

2. Two progressively type-II censored samples with given censoring scheme $(1,0,2,1,0,3,0,2,0,1)$ from GPD are generated. Taking the number of observed failures is $\mathrm{r}=7$ and 8 , the $90 \%$ and $95 \%$ BPI for the future $x_{s: n}, s=8,9$ and 10 are constructed, these are given in Table 5 .

\section{CONCLUDING REMARKS}

Based on the one-sample scheme, Bayesian prediction interval is presented in this paper based on a sample of GOS from the GPD. Results based on progressively type II censored and upper RVs are obtained. From the results we may observe

1. From the Tables one can observe that, the BPI increase when the value of the parameter $\sigma$ increase, and that is because the observation values from the GPD are bounded by $\sigma$, this leads to when $\sigma$ has a small value, the data will be in a small range and thus minimize the length of the BPI.

2. Tables show that the lengths of BPI are sensitive to the size of known observations, i.e, increasing the available information leads to shrinking the intervals. Noted that when confidence level increases the length of intervals also increases.

3. The width of the BPI increases by increasing $s$, i.e, the width of the BPI for a specific future observation is wider than the previous one when other parametric values are fixed.

4. It has been observed from the Tables 4 and 5 that, all the properties of the BPI in case of unknown two 
parameters are similar to the case when one parameter is known.

5. Different values of both of the prior parameters and sample size rather than those appeared in the Tables have been considered but did not change the previous conclusion.

Table 1: $90 \%$ and $95 \%$ BPI for future progressive type II censoring observations $x_{s}$ in case of FSS

\begin{tabular}{|c|c|c|c|c|c|}
\hline \multirow{2}{*}{$\boldsymbol{r}$} & \multirow{2}{*}{$s$} & \multicolumn{2}{|c|}{$\sigma=0.5$} & \multicolumn{2}{|c|}{$\sigma=0.1$} \\
\hline & & 90\% BPI (Width) & 95\% BPI (Width) & 90\% BPI (Width) & 95\% BPI (Width) \\
\hline \multirow[t]{3}{*}{7} & 8 & $0.0086,0.0189(0.0103)$ & $0.0086,0.0218(0.0132)$ & $0.0013,0.0032(0.0019)$ & $0.0023,0.0053(0.003)$ \\
\hline & 9 & $0.0099,0.0344(0.0245)$ & $0.0094,0.0408(0.0313)$ & $0.0016,0.0061(0.0045)$ & $0.0015,0.0073(0.0058)$ \\
\hline & 10 & $0.0126,0.0552(0.0425)$ & $0.0115,0.0653(0.0537)$ & $0.0021,0.0100(0.0079)$ & $0.0019,0.0119(0.0100)$ \\
\hline \multirow[t]{2}{*}{8} & 9 & $0.0120,0.0320(0.0199)$ & $0.0119,0.0376(0.0256)$ & $0.0016,0.0051(0.0034)$ & $0.0016,0.0060(0.0044)$ \\
\hline & 10 & $0.0142,0.0529(0.0386)$ & $0.0134,0.0621(0.0487)$ & $0.0020,0.0087(0.0067)$ & $0.0018,0.0103(0.0084)$ \\
\hline
\end{tabular}

Table 2: 90\% and 95\% BPI for progressive type-II censoring observation $x_{s}$ in case of Poisson RSS.

\begin{tabular}{|cccccc|}
\hline \multirow{2}{*}{$\boldsymbol{r}$} & $\boldsymbol{s}$ & \multicolumn{2}{|c}{$\boldsymbol{\sigma}=\mathbf{0 . 5}$} \\
\cline { 3 - 7 } & & $\mathbf{9 0 \%}$ BPI (Width) & $\mathbf{9 5 \%}$ BPI (Width) & $\mathbf{9 0 \%}$ BPI (Width) & 95\% BPI (Width) \\
\hline $\mathbf{7}$ & $\mathbf{8}$ & $0.3241,0.3378(0.0136)$ & $0.3240,0.3416(0.0175)$ & $0.0723,0.0745(0.0021)$ & $0.0723,0.0751(0.0027)$ \\
& $\mathbf{9}$ & $0.3384,0.3548(0.0164)$ & $0.3374,0.3597(0.0222)$ & $0.0746,0.0771(0.0025)$ & $0.0744,0.0779(0.0034)$ \\
& $\mathbf{1 0}$ & $0.3548,0.3761(0.0212)$ & $0.3554,0.3819(0.0265)$ & $0.0772,0.0805(0.0033)$ & $0.0772,0.0814(0.0041)$ \\
\hline $\mathbf{9}$ & $\mathbf{1 0}$ & $0.2212,0.2318(0.0105)$ & $0.2212,0.2347(0.0135)$ & $0.0618,0.0637(0.0019)$ & $0.0618,0.0642(0.0024)$ \\
\hline
\end{tabular}

Table 3: 90\% and 95\% BPI for future upper record observations

\begin{tabular}{|cccccc|}
\hline \multirow{r}{*}{$\boldsymbol{r}$} & $\boldsymbol{s}$ & \multicolumn{2}{|c|}{$\boldsymbol{\sigma}=\mathbf{0 . 5}$} \\
\cline { 3 - 7 } & & $\mathbf{9 0 \%}$ BPI (Width) & $\mathbf{9 5 \%}$ BPI (Width) & $\mathbf{9 0 \%}$ BPI (Width) & 95\% BPI (Width) \\
\hline $\mathbf{7}$ & $\mathbf{8}$ & $0.2711,0.3454(0.0742)$ & $0.2705,0.3622(0.0917)$ & $0.0692,0.0826(0.0133)$ & $0.0691,0.0852(0.0161)$ \\
& $\mathbf{9}$ & $0.2783,0.3808(0.1025)$ & $0.2756,0.3983(0.1226)$ & $0.0706,0.0880(0.0174)$ & $0.0701,0.0905(0.0203)$ \\
& $\mathbf{1 0}$ & $0.2883,0.4064(0.1181)$ & $0.2838,0.4232(0.1393)$ & $0.0725,0.0916(0.0190)$ & $0.0717,0.0937(0.0219)$ \\
\hline $\mathbf{8}$ & $\mathbf{9}$ & $0.2882,0.3538(0.0656)$ & $0.2876,0.3686(0.0810)$ & $0.0714,0.0830(0.0116)$ & $0.0713,0.0854(0.0141)$ \\
& $\mathbf{1 0}$ & $0.2946,0.3855(0.0908)$ & $0.2922,0.4008(0.1086)$ & $0.0726,0.0880(0.0153)$ & $0.0722,0.0902(0.0180)$ \\
\hline
\end{tabular}

Table 4: 90\% and 95\% BPI for future upper record observations $x_{s}$ when unknown $\theta$ and $\sigma$.

\begin{tabular}{|cccccc|}
\hline \multirow{2}{*}{$\boldsymbol{r}$} & $\boldsymbol{s}$ & \multicolumn{2}{c}{$\theta=\mathbf{0 . 0 1 5 5}$ and $\boldsymbol{\sigma}=\mathbf{0 . 2 2 5}$} & \multicolumn{2}{c|}{$\boldsymbol{\theta = 0 . 0 1 5 5 \text { and } \boldsymbol { \sigma } = \mathbf { 0 . 5 8 6 }}$} \\
\cline { 3 - 7 } & & $\mathbf{9 0 \%}$ BPI (Width) & $\mathbf{9 5 \%}$ BPI (Width) & $\mathbf{9 0 \%}$ BPI (Width) & $\mathbf{9 5 \%}$ BPI (Width) \\
\hline $\mathbf{7}$ & $\mathbf{8}$ & $0.0177,0.0281(0.0104)$ & $0.0176,0.0313(0.0136)$ & $0.1074,0.1429(0.0354)$ & $0.1071,0.1522(0.0450)$ \\
& $\mathbf{9}$ & $0.0185,0.0351(0.0165)$ & $0.0182,0.0394(0.0212)$ & $0.1106,0.1646(0.0539)$ & $0.1047,0.1763(0.0716)$ \\
& $\mathbf{1 0}$ & $0.0196,0.0415(0.0218)$ & $0.0191,0.0469(0.0278)$ & $0.1154,0.1844(0.0690)$ & $0.1133,0.1986(0.0853)$ \\
$\mathbf{8}$ & $\mathbf{9}$ & $0.0185,0.0275(0.0090)$ & $0.0184,0.0305(0.0117)$ & $0.1167,0.1511(0.0343)$ & $0.1164,0.1600(0.0435)$ \\
& $\mathbf{1 0}$ & $0.0192,0.0335(0.0142)$ & $0.0189,0.0372(0.0182)$ & $0.1132,0.1720(0.0588)$ & $0.1140,0.1832(0.0692)$ \\
\hline
\end{tabular}

Table 5: $90 \%$ and $95 \%$ BPI for future progressive type II censoring observations $x_{s}$ when unknown $\theta$ and $\sigma$.

\begin{tabular}{|c|c|c|c|c|c|}
\hline \multirow{2}{*}{$r$} & \multirow{2}{*}{$s$} & \multicolumn{2}{|c|}{$\theta=0.0155$ and $\sigma=0.726$} & \multicolumn{2}{|c|}{$\theta=0.0155$ and $\sigma=0.184$} \\
\hline & & 90\% BPI (Width) & 95\% BPI (Width) & 90\% BPI (Width) & 95\% BPI (Width) \\
\hline \multirow[t]{3}{*}{7} & 8 & $0.0085,0.0155(0.007)$ & $0.0085,0.0174(0.0089)$ & $0.0020,0.0047(0.0026)$ & $0.0020,0.0054(0.0034)$ \\
\hline & 9 & $0.0095,0.0261(0.0166)$ & $0.0091,0.0300(0.0208)$ & $0.0023,0.0088(0.0064)$ & $0.0022,0.0105(0.0082)$ \\
\hline & 10 & $0.0115,0.0403(0.0288)$ & $0.0107,0.0740(0.0633)$ & $0.0030,0.014(0.0114)$ & $0.0027,0.0173(0.0145)$ \\
\hline \multirow[t]{2}{*}{8} & 9 & $0.0107,0.0245(0.0137)$ & $0.0106,0.0281(0.0175)$ & $0.0021,0.0067(0.0046)$ & $0.0020,0.0080(0.0059)$ \\
\hline & 10 & $0.0123,0.0389(0.0266)$ & $0.0112,0.0449(0.0331)$ & $0.0026,0.0117(0.0091)$ & $0.0024,0.0141(0.0116)$ \\
\hline
\end{tabular}




\section{REFERENCES}

[1] order statistics. Ann. Statist. 3, 119-131.

[2] Castillo, E., Hadi, A. S., 1977. Fitting the generalized Pareto distribution to data. J. Amer Statist. Assoc. 92(440), 1609- 1620.

[3] Kamps, U., 1995. A Concept of Generalized Order statistics. Teubner, Stuttgart, Germany.

[4] Cramer, E. \& Kamps, U., 2003. Marginal distributions of sequential and generalized order statistics. Metrika. 58, 293-310.

[5] Ahmad, A.A., Fawzy, M., 2003. Recurrence relations for single moments of generalized order statistics from doubly truncated distributions and its characterizations. J. Statist. Plann.Inf. 177, 241-249.

[6] Cramer, E., Kamps, U., 2000. Relations for expectations of functions of generalized order statistics. J. Statist. Plann.Inf. 89, 79-89.

[7] Mohie El-Din,M.M, Abdel-Aty, Y., Shafay, A.R., 2011.Two sample Bayesian prediction intervals for order statistics based on the inverse exponential-type distributions using right censored sample. Egyptian Mathematical Society. 19, 102-105.

[8] Prakash, G., 2012. Central Coverage Bayes Prediction Intervals for the Generalized Pareto Distribution. Statistics Research Letters. 1(1), 1-5.

[9] Gupta, D., Gupta, R. C., 1984. On the distribution of order statistics for a random sample size. Statist. Nearland. 38, 13- 19

[10] Kamps, U., Gather, U., 2001. On distributions of generalized order statistics. Statistics. 35, 269-280.

[11] Balakrishnan, N., Aggarwala, R., 2000. Progressive Censoring: Theory, Methods and Applications. Birkh"auser, Boston, Berlin.

[12] Balakrishnan, N., Sandhu, R. A., 1995. A simple simulation algorithm for generating progressive Type-II censored samples. Amer.Statist. 49(2), 229-230. 